\title{
LA MÉTAPHORE
}

\section{PRÉSENTATION DU DOSSIER}

\author{
Jean-Pierre Meunier ${ }^{1}$
}

La métaphore est bien plus qu'un simple jeu de mots né de l'imagination rhétorique, bien plus que la part ludique des échanges verbaux. Elle est sans doute cela, mais elle concerne aussi des aspects plus profonds et plus complexes de la communication. C'est en tout cas ce que donnent à penser divers auteurs contemporains qui, reprenant, après mille autres, l'analyse de la notion dans de nouvelles perspectives, tentent d'en dégager les implications dans les domaines de la pensée, de l'action, de la culture en général. C'est ainsi que, dans le paysage théorique relatif à la communication, la notion de métaphore, peu à peu, quitte les marges pour se rapprocher du centre. D'une part, en effet, la métaphore semble se manifester et produire des effets dans toutes les dimensions de la communication successivement mises au jour par la recherche: la signification, la relation, la cognition. D'autre part, bien loin de limiter son action aux seuls aspects proprement linguistiques de la communication, elle semble au contraire travailler toutes les "matières signifiantes" que la sémiologie a incluses dans son domaine d'investigation: la matière

1 Professeur au Département de communication de l'Université catholique de Louvain et Président du Département.

Recherches en communication, $\mathrm{n}^{\circ} 1$ (1994). 
iconique, la matière sonore, la matière vocale... Ce sont d'ailleurs ces matières typiquement non-verbales qui semblent constituer le lieu de prédilection de la métaphore.

Examinons brièvement les trois dimensions de la communication énumérées plus haut.

Sur le plan de la signification, le concept de métaphore -généralement associé à celui de métonymie- est apparu fondamental dès lors qu'il a fallu comprendre et expliquer les processus de production et de lecture du sens contenu dans les images. Il faut se souvenir ici de la première sémiologie, de la Rhétorique de l'image de Barthes, des travaux de Metz sur la signification au cinéma, et d'autres. Par choc en retour, du reste, ces recherches ont suscité de nouvelles interrogations sur le sens verbal lui-même: la métaphore ne serait-elle pas un des principaux processus producteurs de sens dans le langage verbal?

Sur le plan de la relation, aspect de la communication devenu saillant dans le cadre de la perspective pragmatique, la notion de métaphore n'a peut-être pas acquis les mêmes prérogatives qu'en sémiologie de l'image. On a cependant noté à maintes reprises que la rhétorique -dont la métaphore est un instrument- est fondamentalement liée à un type particulier de communication performative -la communication persuasive- et que, de fait, la métaphore est omniprésente dans la publicité et la propagande. Par ailleurs, et plus important encore à considérer, la métaphore, on vient de le voir, est essentielle à la communication non verbale -justement nommée communication analogique- et l'on sait depuis les travaux de Bateson que celle-ci est constitutive de la relation de communication. Il reste cependant beaucoup à découvrir au sujet de cette double liaison: métaphore-analogie-relation ${ }^{1}$.

Sur le plan de la cognition, le concept de métaphore a accompli -il est tentant d'utiliser ici une métaphore guerrière- une percée remarquable, notamment avec les travaux de Lakoff et Johnson ${ }^{2}$. Pour ces auteurs, la métaphore n'est pas seulement affaire de langage, elle concerne avant tout la pensée et l'action en ce sens que, structurant notre système conceptuel ordinaire, elle structure du même coup nos

1 A ce sujet, des travaux tels que ceux que Fonagy a consacrés à l'étude du style vocal ouvrent une perspective tout à fait intéressante (Ivan FONAGY, La vive voix. Essai de psycho-phonétique, Paris, Payot, 1983).

2 Cf. notamment: George LAKOFF et Mark JoHnson, Les métaphores dans la vie quotidienne, Paris, Éditions de Minuit, 1985. 
manières de voir et d'agir. Or, comme par ailleurs, dans la ligne de certains travaux issus de Grice, Searle, Sperber et Wilson ${ }^{1}$, etc., la communication apparaît maintenant indissociable de la cognition, on voit se constituer une nouvelle triade conceptuelle (communicationcognition-métaphore) réclamant l'attention des chercheurs.

Ainsi reliée, mais par des liens encore relativement imprécis, à tous les aspects connus de la communication, la métaphore se présente à la fois comme un concept élucidant et comme un concept à élucider, comme un concept capable d'éclairer toutes sortes de relations concrètes de communication et devant recevoir en retour de ce qu'il éclaire les éléments propres à l'éclairer lui-même, en tant que concept abstrait; démarche circulaire entre le concret et l'abstrait, entre l'observation et la théorie. Un tel concept devait s'imposer comme thème privilégié à une revue dont le projet est précisément de fournir, à propos de concepts riches en signification sur le plan de la communication, des dossiers ouverts à des interrogations et à des recherches à la fois théoriques et empiriques, abstraites et concrètes, générales et sectorielles. On trouvera dans ce dossier -du moins l'espérons-nous- de quoi alimenter ce va-et-vient abstrait-concret que l'on vient d'évoquer.

"La métaphore est au cour de la connaissance comme de la relation intersubjective" écrit D. Bougnoux dans l'article d'ouverture, soulignant ainsi d'emblée la dimension réticulaire du concept et l'ampleur du champ d'investigation. La métaphore n'est pas seulement dans la poésie explique-t-il encore, elle réside aussi dans la science, malgré les efforts que déploie celle-ci pour élaguer le sens des mots. Ce serait précisément une des tâches des études de communication de faire apparaitre les liens entre langage poétique et langage scientifique.

Les deux contributions suivantes se situent sur le plan des définitions abstraites et cherchent, chacune à leur manière, à proposer une élucidation du concept.

Le texte de $\mathrm{Ph}$. Verhaegen situe la métaphore dans le cadre de la sémiotique de Peirce, la faisant apparaître dès lors comme une variété d'icône. Cette démarche présente l'avantage de décentrer la réflexion sur la métaphore relativement au domaine des faits de langage et de

1 Cf, notamment Dan SPERBER et Deirdre WILSON, La pertinence: communication et cognition, Paris, Éditions de Minuit, 1989. 
permettre ainsi d'élucider certains aspects de son fonctionnement cognitif spécifique.

Avec le texte de $M$. Baudoux, on réintègre le domaine particulier des échanges linguistiques, mais c'est pour l'aborder -au travers de l'ouvrage de M. Prandi, Grammaire philosophique des tropes ${ }^{1}$, dont M. Baudoux nous présente un commentaire éclairant- dans le cadre d'une linguistique enrichie de considérations issues de notre expérience du monde et d'autrui, c'est-à-dire de notre capacité de comprendre les choses avec des mots et de vouloir les communiquer. Il s'ensuit une série de définitions et distinctions permettant de relier la métaphore aux différentes notions -interprétation, inférence, modèles...- dont la rencontre est à la base de l'élaboration progressive d'un nouveau modèle théorique de la communication fondée sur la cognition.

Les textes qui suivent cette copieuse entrée en matière théorique s'intéressent davantage aux aspects culturels des métaphores, c'est-àdire aux implications concrètes dans la vie quotidienne, où pensée et pratique se trouvent intimement liées, des métaphores concrètes qui modèlent nos représentations et orientent nos actions.

Le texte de J.-P. Meunier tente de mesurer les conséquences pour les communications réelles des métaphores sous-jacentes aux théories qui se donnent précisément pour but de dire ce que sont les communications. Les articles de A. Gryspeerdt et Ph. Charlier tentent de leur côté de saisir le jeu des métaphores dans la vie des organisations, le premier en tentant de préciser le fonctionnement d'une métaphore courante dans la communication d'entreprise -la métaphore de l'ambassadeur-, le second en tentant de creuser les effets -dans la représentation, le vécu, la relation...- des diverses métaphores par lesquelles les organisations prennent sens pour leurs membres. Enfin, l'article de G. Gauthier nous entraîne sur le terrain de la communication politique et nous convie à nous interroger sur la présence, au sein du discours politique, de métaphores guerrières.

Le thème de la métaphore est riche, si riche que Recherches en communication n'a pu faire autrement, pour faire droit à la diversité des réflexions suscitées, que d'y consacrer son second numéro. On y poursuivra dans la voie ouverte ici, c'est-à-dire qu'on y trouvera des textes consacrés à l'explicitation théorique -on y découvrira notam-

1 Michele Prandi, Grammaire philosophique des tropes, Paris, Éditions de Minuit, 1992, 252 p. 
ment une approche des rapports entre narration et métaphore- et des textes centrés sur l'observation du jeu des métaphores dans la vie sociale et culturelle. Ce seront cependant d'autres secteurs et aspects de cette vie qui y seront considérés: la presse, la communication politique, la communication pédagogique... Ainsi espérons-nous nous mettre en mesure d'effectuer un autre va-et-vient productif entre l'abstrait et le concret. 